\title{
Endosialin/CD248 may be a potential therapeutic target to prevent the invasion and metastasis in osteosarcoma
}

\author{
YUMIKO KONDO $^{1}$, KANYA HONOKI $^{1,2}$, SHINGO KISHI $^{1,3}$, SHIORI MORI $^{3}$, RINA FUJIWARA-TANI ${ }^{3}$, \\ SHINJI TSUKAMOTO ${ }^{1,4}$, HIROMASA FUJII ${ }^{1}$, HIROKI KUNIYASU ${ }^{3}$ and YASUHITO TANAKA ${ }^{1}$ \\ Departments of ${ }^{1}$ Orthopedic Surgery, ${ }^{2}$ Orthopedic Oncology and Reconstructive Medicine, ${ }^{3}$ Molecular Pathology and \\ ${ }^{4}$ Rehabilitation Medicine, Nara Medical University, Kashihara, Nara 634-8521, Japan
}

Received April 17, 2021; Accepted September 24, 2021

DOI: $10.3892 / \mathrm{ol} .2021 .13160$

\begin{abstract}
Endosialin/CD248/tumor endothelial marker 1 is classified as a C-type lectin-like transmembrane receptor, found on the plasma membrane of activated mesenchymal cells, which binds to fibronectin. Although endosialin is expressed at high levels in stem-like cells of sarcomas, its role has not been fully uncovered. The present study aimed to determine whether endosialin expression is associated with tumor progression and metastasis, and whether endosialin has the potential to act as a novel therapeutic target in osteosarcoma (OS) using MORAb-004/ontuxizumab, a humanized monoclonal antibody, which targets the type $\mathrm{C}$ lectin domain of endosialin. The results demonstrated that endosialin was highly expressed in OSs with metastatic disease. Furthermore, MORAb-004 had no cytostatic effect on OS cells in vitro and did not change the expression of stem cells and differentiation markers; however, it inhibited migration of OS cells. Taken together, these results suggest that endosialin may play a role in migration, and may be involved in the metastatic process of OSs. Furthermore, MORAb-004 reduces the motility of OS cells, and suppresses invasion and the development of metastatic lesions.
\end{abstract}

\section{Introduction}

Endosialin/CD248/tumor endothelial marker 1 (TEM1) is classified as a C-type lectin-like transmembrane receptor, found on the plasma membrane of activated mesenchymal cells, which binds to fibronectin (FN) $(1,2)$. Endosialin expression is essentially limited to embryonal development and is expressed at very low levels in normal adult tissues; however, it is upregulated in pathological states, including tumor progression and metastasis. Endosialin does not function as an active metastasis promoting signaling factor in tumor cells to gain

Correspondence to: Professor Kanya Honoki, Department of Orthopedic Oncology and Reconstructive Medicine, Nara Medical University, 840 Shijo-cho, Kashihara, Nara 634-8521, Japan

E-mail:kahonoki@naramed-u.ac.jp

Key words: osteosarcoma, endosialin, invasion, metastasis invasive behavior, but rather as a facilitator, tethering, tumor cells to the matrix and actively mediating their transmigration through the vascular basement membrane and underlying endothelial monolayer (3). In sarcomas, endosialin expression is found in malignant tumor cells, perivascular and stromal cells, and is speculated to be involved in tumor angiogenesis (4). Another study reported that endosialin-expressing stem-like cells of osteosarcoma (OS) possess self-renewing and invasive properties, and are highly drug resistant. Although endosialin is highly expressed in stem-like cells of OSs (5), its role has not been fully uncovered.

MOR Ab-004/ontuxizumab, a humanized monoclonal antibody, targets the type C lectin domain of endosialin (4). In preclinical studies, binding of ontuxizumab to two paired cell lines (Ewing's and synovial sarcoma cell lines) was confirmed via semi-quantitative immunofluorescence (6). In addition, immunofluorescent staining of ontuxizumab-treated human pericytes exhibited cellular internalization of the antibody, with a corresponding reduction of surface endosialin. MORAb-004 treated tumors display overall shortened and distorted blood vessels. The CD248 levels on cell surfaces of neovasculature pericytes are significantly reduced due to its internalization. This reduction of CD248 is also accompanied by reduced $\alpha$-SMA expression, depolarization of pericytes and endothelium, and ultimately dysfunctional microvessels. These findings suggest that MORAb-004 reduces CD248 on pericytes, impairs tumor microvasculature maturation and ultimately suppresses tumor development (7). The phase II, randomized controlled trial of MORAb-004/ontuxizumab was performed to evaluate the safety, as well as the efficacy of ontuxizumab with the combination of gemcitabine and docetaxel $(\mathrm{G} / \mathrm{D})$ in metastatic soft tissue sarcoma $(8,9)$. This study bases on the hypothesis that blocking endosialin-mediated tumor angiogenesis with ontuxizumab can enhance the efficacy of G/D in sarcomas. However, ontuxizumab with G/D exhibited no enhanced activity compared with chemotherapy alone in soft tissue sarcomas, whereas the safety of the combination was consistent with G/D alone. Preclinical experiments in wild-type and endosialin-deficient mice indicate that stromal endosialin does not affect primary tumor growth but strongly promotes metastasis (3).

The present study aimed to determine whether endosialin expression is associated with tumor progression and metastasis 
in OS, and whether endosialin has the potential to be a novel therapeutic target via MORAb-004/ontuxizumab.

\section{Materials and methods}

Human OS specimens. A total of 18 clinical specimens were collected from patients with OS via biopsy at the Department of Orthopedic Surgery, Nara Medical University (Kashihara, Japan) prior to any treatments, including surgery, chemotherapy and radiotherapy, between December 1991 and July 2017. A total of 18 patients with osteosarcoma were included in the present study, including 12 men and six women. The median age of all patients was 23 years (age range, 8-69 years). Only specimens biopsied for the initial diagnosis were selected, those at the time of recurrence and during or after treatments were excluded. Specimens were stored at $4^{\circ} \mathrm{C}$ until subsequent experiments. The present study was approved by the Nara Medical University Certified Review Board (approval no. 2833) and written informed consent was provided by all patients or family members prior to the study start. Informed consent was provided in the form of opt-out on the website (https://naraseikei.com/patients). Patient characteristics are presented in Table I.

Immunohistochemistry (IHC). Specimens were formalin-fixed and paraffin-embedded immediately after the biopsy. Consecutive $4 \mu \mathrm{m}$ sections were cut from each block, and underwent immunohistochemical staining analysis for CD248. IHC analysis was performed via the immunoperoxidase technique, as previously described (10). Briefly, following antigen retrieval with autoclave treatment in citrate buffer for $15 \mathrm{~min}$ at $121^{\circ} \mathrm{C}$, specimens were incubated with $3 \% \mathrm{H}_{2} \mathrm{O}_{2}$-methanol for 10 min and subsequently blocked with PBS containing $2 \%$ skimmed milk for $30 \mathrm{~min}$ at room temperature. Following incubation with primary antibody against CD248 $(2 \mu \mathrm{g} / \mathrm{ml}$; 1:100 dilution; cat. no. HPA051856; Cosmo Bio Co., Ltd.) at room temperature for $2 \mathrm{~h}$, specimens were briefly washed with PBS and incubated with secondary antibody conjugated with peroxidase (1:100 dilution; cat. no. K4061; Envision ${ }^{\mathrm{TM}}+$ Dual Link System-HRP; Dako; Agilent Technologies, Inc.) at room temperature for $1 \mathrm{~h}$. The specimens were re-washed with PBS, stained with DAB (Dako; Agilent Technologies, Inc.) for $5 \mathrm{~min}$ at room temperature and subsequently washed lightly in distilled water and running water. The slides were counterstained with Meyer-hematoxylin for $1 \mathrm{~min}$ at room temperature, washed in phosphate buffer for $5 \mathrm{~min}$, running water for $10 \mathrm{~min}$, dehydrated through graded alcohols, cleared in xylene and cover slipped.

A total of two experienced pathologists evaluated the IHC staining to avoid bias. To assess CD248 expression in IHC staining, staining intensity and staining area (\%) were measured at two hot spots in each specimen using an optical microscope (BX50; x200 magnification; Olympus Corporation). CD248 staining intensity was scored as follows: 0, negative; $1+$, weak; $2+$, moderate and $3+$, strong. The staining intensity of endothelium was defined as $2+$ on the basis that CD248 is a tumor endothelium marker, which is expressed in vascular endothelial cells of malignant tumors (11). The staining area $(\%)$ at each staining intensity was measured using ImageJ version 1.52 software (12). The CD248 positive expression score (IHC score) was calculated as the sum of staining intensity and staining area (\%), which ranged from 0-300. The mean IHC score in each specimen was used for statistical analysis. CD248 IHC score was determined via receiver operating characteristic (ROC) curve analysis to determine the cut-off value, and Fisher's exact test was used to assess the association between CD248 expression and metastasis. An IHC score of CD248 $\geq 20.5$ was considered positive, while an IHC score $<20.5$ was negative, using the cut-off value.

Cell culture and reagents. The OS cell lines, SaOS2, U2OS, HOS and MG63, were purchased from the American Type Culture Collection and maintained in (DMEM; Sigma-Aldrich; Merck KGaA) supplemented with $10 \%$ fetal bovine serum (FBS; Sigma-Aldrich; Merck KGaA) and $50 \mathrm{U} / \mathrm{ml}$ penicillin/streptomycin (Nacali Tesque Inc.) at $37^{\circ} \mathrm{C}$ with $5 \% \mathrm{CO}_{2}$.

FN was purchased from FUJIFILM Wako Pure Chemical Corporation, while MORAb-004/ontuxizumab was purchased from Eizai Inc. (https://us.eisai.com).

Cell viability assay. Cells (SaOS2, U2OS and MG63) were seeded into 12-well pates at a density of 10,000 cells/well. The effects of MORAb-004 (0, 5, 10 and $20 \mu \mathrm{mol} / \mathrm{l})$ and FN ( 0 and $10 \mu \mathrm{g} / \mathrm{ml}$ ) on cell proliferation were assessed via the MTS assay after $48 \mathrm{~h}$, as previously described (13). The MTS assay was (Promega Corporation) performed according to the manufacturer's instructions. Briefly, MTS solution was added to each well for $2 \mathrm{~h}$ at $37^{\circ} \mathrm{C}$ with $5 \% \mathrm{CO}_{2}$, and the absorbance was measured at a wavelength of $490 \mathrm{~nm}$, using a microplate reader (Multiskan FC; Thermo Fisher Scientific Inc.).

Apoptosis analysis. Apoptosis was assessed in $\mathrm{SaOS}$ and U2OS cells via (Sigma-Aldrich; Merck $\mathrm{KGaA}$ ) at room temperature for $10 \mathrm{~min}$, with MORAb-004 (0 or $20 \mu \mathrm{mol} / \mathrm{l})$ and FN (0 or $10 \mu \mathrm{g} / \mathrm{ml}$ ) for $48 \mathrm{~h}$. Apoptotic cells were observed under a fluorescence microscope (x200 magnification; Keyence Corporation).

Reverse transcription-quantitative (RT-q)PCR. RT-qPCR analysis was performed to assess the effect of MORAb-004 with FN on the expression levels of stem cell markers: Octamer-binding transcription factor-3 (OCT3), Kruppel-like factor 4 (KLF4), nucleostemin (NS) and clusters of differentiation 44 (CD44), and differentiation markers: Alkaline phosphatase (ALP), Collagen $1 \alpha(\mathrm{COL} 1 \alpha)$ and runt-related transcription factor 2 (RUNX2), in SaOS2 and U2OS cells. A total of $0.5 \mu \mathrm{g}$ total RNA was extracted from ontuxizumab ( 0 or $20 \mu \mathrm{mol} / \mathrm{l})$ with FN $(10 \mu \mathrm{g} / \mathrm{ml})$-treated and untreated (as a control) cells using TRIzol ${ }^{\circledR}$ RNA Isolation Reagents (Thermo Fisher Scientific, Inc.) and the RNeasy kit (Qiagen $\mathrm{GmbH}$ ), followed by cDNA synthesis. In addition, the expression of endosialin/human TEM1 in OS cell lines was assessed via RT-qPCR analysis. A total of $0.5 \mu \mathrm{g}$ of total RNA was extracted from ontuxizumab $(20 \mu \mathrm{mol} / \mathrm{l})$-treated cells as aforementioned. The primer sequences used for qPCR were synthesized by Sigma-Aldrich; Merck KGaA and are listed in Table II. The following thermocycling conditions were used: $95^{\circ} \mathrm{C}$ for $5 \mathrm{~min} ; 40$ cycles at $95^{\circ} \mathrm{C}$ for $30 \mathrm{sec}, 63.9-71^{\circ} \mathrm{C}$ for $30 \mathrm{sec}$ and $72^{\circ} \mathrm{C}$ for $30 \mathrm{sec}$. PCR products were electrophoresed using a $2 \%$ agarose gel and stained with ethidium bromide for $20 \mathrm{~min}$ at room temperature with shading. $\beta$-actin mRNA was 


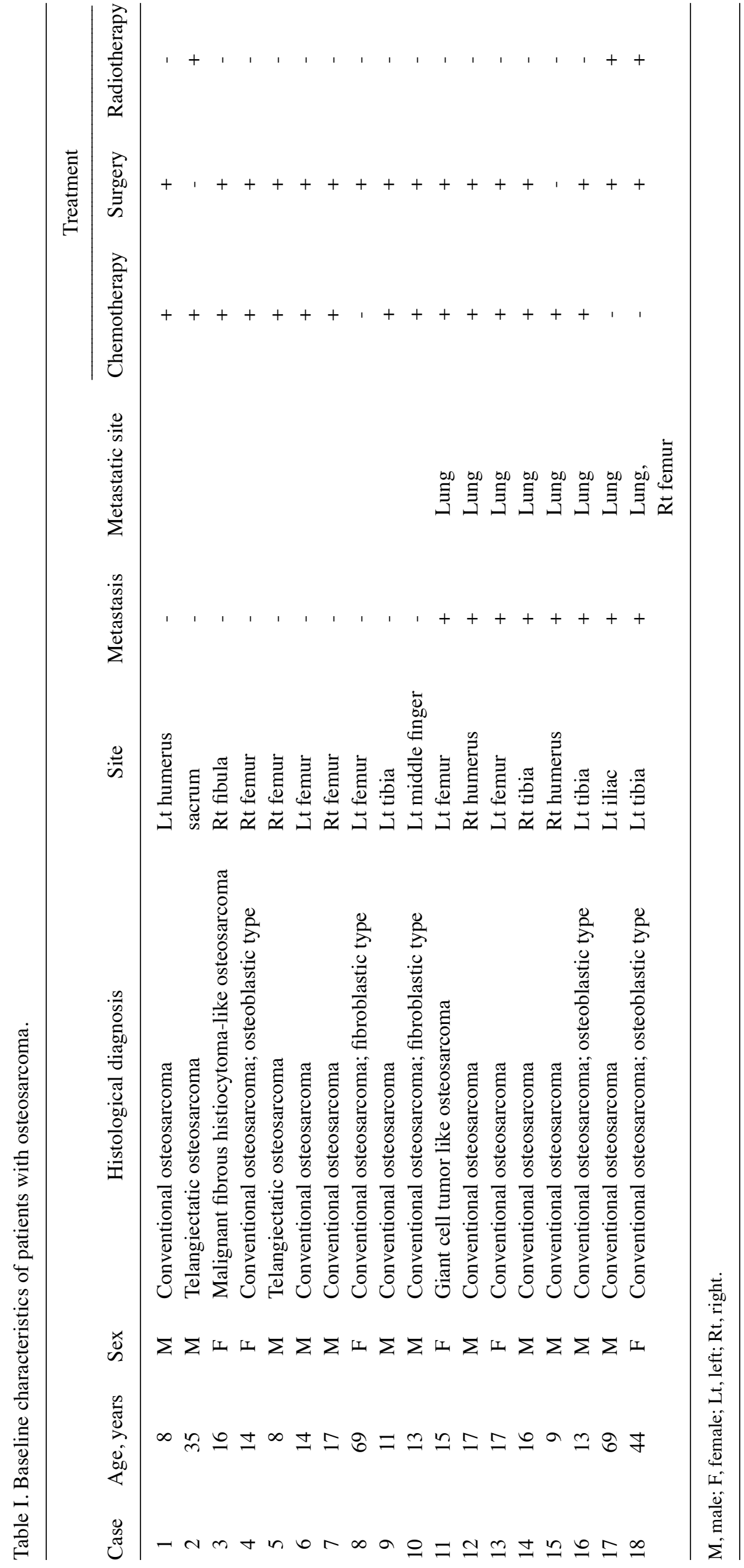


Table II. Primer sequences used for quantitative PCR.

\begin{tabular}{ll}
\hline Gene & \multicolumn{1}{c}{ Primer sequence (5'-3') } \\
\hline OCT3 & F GAAGGATGTGGTCCGAGTGT \\
KLF4 & F: GTGAAGTGAGGGCTCCCATA \\
& R: ATCTTTCTCCACGTTCGCGTCTG \\
NS & F: ATTGCCAACAGTGGTGTTCA \\
& R: AATGGCTTTGCTGCAAGTTT \\
CD44 & F: AAGGTGGAGCAAACACAACC \\
ALP & R: AGCTTTTCTTCTGCCCACA \\
& F: CGCCTACCAGCTCATGCATA \\
COL1 $\alpha$ & R: GCTCTTCCAGGTGTCAACGA \\
& F: CAGGCTGGTGTGATGGGATT \\
RUNX2 & R: GGGCCTTGTTCACCTCTCTC \\
& F: GCGCATTCCTCATCCCAGTA \\
hTEM1 & R: GGCTCAGGTAGGAGGGGTAA \\
& F: GGACACAGATGAGTGCCAGA \\
& R: CAGGCCTCGTCTTCATCTTC
\end{tabular}

F, forward; R, reverse; OCT3, octamer-binding transcription factor-3; KLF4, Kruppel-like factor 4; NS, nucleostemin; CD44, clusters of differentiation 44; ALP, alkaline phosphatase; COL1 $\alpha$, Collagen $1 \alpha$; RUNX2, runt-related transcription factor 2; hTEM1, human tumor endothelial maker.

also amplified for use as an internal control. For the quantification of PCR products, the band quantification was performed using ImageJ version 1.52 software (National Institutes of Health). The experiments were performed in triplicate.

Western blotting. Western blot analysis was performed on the stem cell markers, CD44V9 and NS, in SaOS2 and U2OS cells, which were treated with ontuxizumab $(20 \mu \mathrm{mol} / \mathrm{l})$ and FN $(10 \mu \mathrm{g} / \mathrm{ml})$ or untreated (as a control). Whole cell lysates were prepared using M-PER ${ }^{\mathrm{TM}}$ Mammalian Protein Extraction Reagent (Thermo Fisher Scientific Inc.). The protein concentrations of cell lysates were evaluated via the Bradford method, using bovine serum albumin as a standard (14). Proteins $(20 \mu \mathrm{g})$ were separated from the cell lysates via SDS-PAGE on $12.5 \%$ gels and electrotransferred onto nitrocellu $\neg$ lose membranes. The membranes were blocked with a solution of $5 \%$ skimmed milk at room temperature overnight, and then incubated with primary antibodies against CD44V9 (1:5,000 dilution; cat. no. 1370435; Seikagaku Corporation) and NS (1:5,000 dilution; cat. no. ab70346; Abcam) for $12 \mathrm{~h}$ at $4^{\circ} \mathrm{C}(15)$. Subsequently, the membranes were washed with PBS and incubated with the secondary antibody, anti-rabbit imunoglobulins/HRP (1:1,000 dilution; cat. no. P0217; Dako; Agilent Technologies Inc.) for $1 \mathrm{~h}$ at room temperature. A tubulin antibody (1:5,000 dilution; cat. no. ab4047; Abcam) was used to measure the amount of protein loaded in each lane. Immune complexes were visualized using the CSA system (Dako; Agilent Technologies, Inc.). The intensities of the band were using ImageJ version 1.52 software.
Wound healing assay. The wound healing assay was performed to assess the effect of MORAb-004 with FN on the migratory ability of OS cell lines (HOS, SaOS2 and U2OS). Cells were seeded into $3.5 \mathrm{~cm}$ culture dishes and treated with MORAb-004 ( 0 or $20 \mu \mathrm{mol} / \mathrm{l})$ and $\mathrm{FN}(10 \mu \mathrm{g} / \mathrm{ml})$. Following incubation with $10 \% \mathrm{FBS}$ at $37^{\circ} \mathrm{C}$ for $24 \mathrm{~h}$, cells grown to sub-confluence were scraped to make a cell-free area with a sharp edge (16-18). Cells migrating into the scraped area was captured at 0 and $24 \mathrm{~h}$ after scraping at x100 magnification under an optical microscope (ECLIPSE Ti-S100; Nikon Corporation, Minato Ward) (16). The width between the edges of each scraped area was measured at 0 and $24 \mathrm{~h}$ and the migration rate (MR) was calculated using the following formula: $\mathrm{MR}(\%)=(\mathrm{Wt}=0-\mathrm{Wt}=24)$ $\mathrm{x} 100 / \mathrm{Wt}=0, \mathrm{Wt}=0$ : the width at $0 \mathrm{~h}, \mathrm{Wt}=24$ : The width at $24 \mathrm{~h}$ after. The width was showed by yellow straight line in each figure. The experiments were performed in triplicate.

Transwell migration assay. The modified Boyden chamber assay was performed to assess in vitro migration of the OS cell lines, SaOS2, U2OS and MG63 treated with MORAb-004. The CytoSelect ${ }^{\mathrm{TM}}$ 24-well Cell Haptotaxis Assay $(8 \mu \mathrm{m})$ kit (FN-coated, Fluorometric; Cell Biolabs Inc.) was used, according to the manufacturer's instructions. $0.75 \times 10^{6}$ cells $/ \mathrm{ml}$ were suspended in $500 \mu \mathrm{l}$ of regular medium (DMEM supplemented with 10\% FBS; Sigma-Aldrich; Merck KGaA) and $2.3 \times 10^{5}$ cells $/ \mathrm{ml}$ were placed in the upper part of the chamber, while the lower part of the chamber was filled with regular medium. Following incubation for $24 \mathrm{~h}$ at $37^{\circ} \mathrm{C}$, the filters were carefully removed from the inserts, stained with hematoxylin for $10 \mathrm{~min}$ at room temperature and mounted on microscopic slides. Stained cells were counted in whole inserts at x100 magnification under an optical microscope (BX50; Olympus Corporation) (16). The area ratio occupied by cells per field of view in the photomicrograph after $24 \mathrm{~h}$ was quantified in the MORAb-004 treated and the untreated groups using ImageJ version 1.52 software (National Institutes of Health), and the untreated group was used as the control. The area ratio of the MORAb-004 treated group based on the control was calculated as the migration rate (\%). The experiments were performed in triplicate.

Statistical analysis. Statistical analysis was performed using SPSS version 26 software (IBM Corp.). Data are presented as the mean \pm SD. IHC analysis was performed once. RT-qPCR, the apoptosis analysis, western blotting, the wound healing assay, the Transwell migration assay were performed in triplicate. Fisher's exact and $\chi^{2}$ tests (two-tailed) were used for IHC analysis. To assess cell viability, RT-qPCR, wound healing assay and apoptosis one-way ANOVA followed by Tukey's post hoc test was used. Unpaired Student's t-test was used to assess Transwell migration assay. $\mathrm{P}<0.05$ (two-sided) was considered to indicate a statistically significant difference.

\section{Results}

Association between endosialin/CD248 expression and metastasis in human OS. A total of 18 human clinical specimens of OS were assessed via IHC analysis to determine the association between endosialin/CD248 expression and metastasis. Fig. 1 depicts endosialin/CD248 expression in human OS tissues. The IHC scores were 5, 7, 55 and 82 in Fig. 1A-D, respectively. 
A
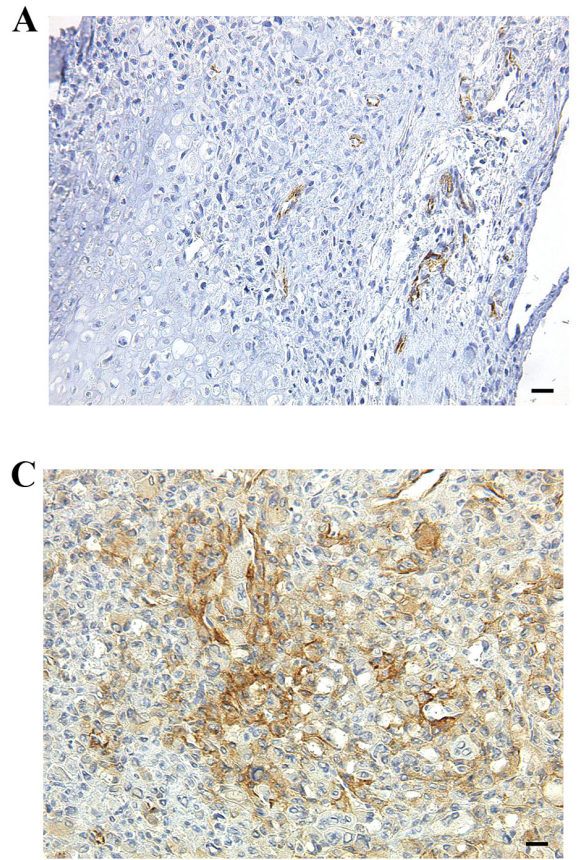

B

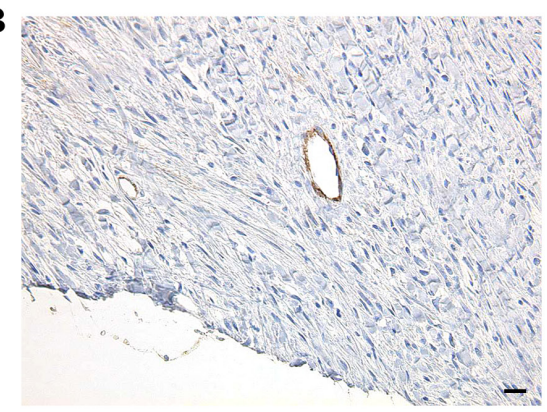

D

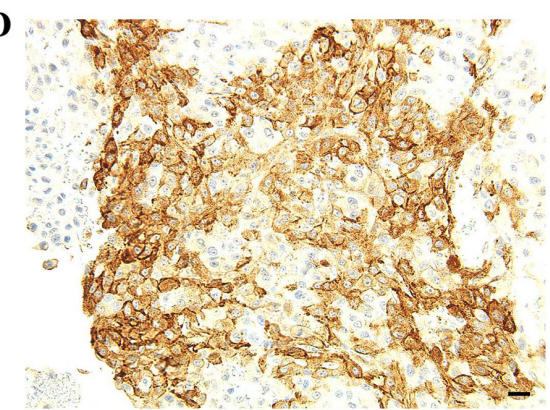

Figure 1. IHC analysis for CD248 expression in OS. (A and B) CD248 expression in non-metastatic cases. (C and D) CD248 expression in metastatic OS. Scale bar, $200 \mu \mathrm{m}$; magnification, x200. IHC scores: (A) 5, (B) 7, (C) 55 and (D) 82. IHC, immunohistochemistry; OS, osteosarcoma.
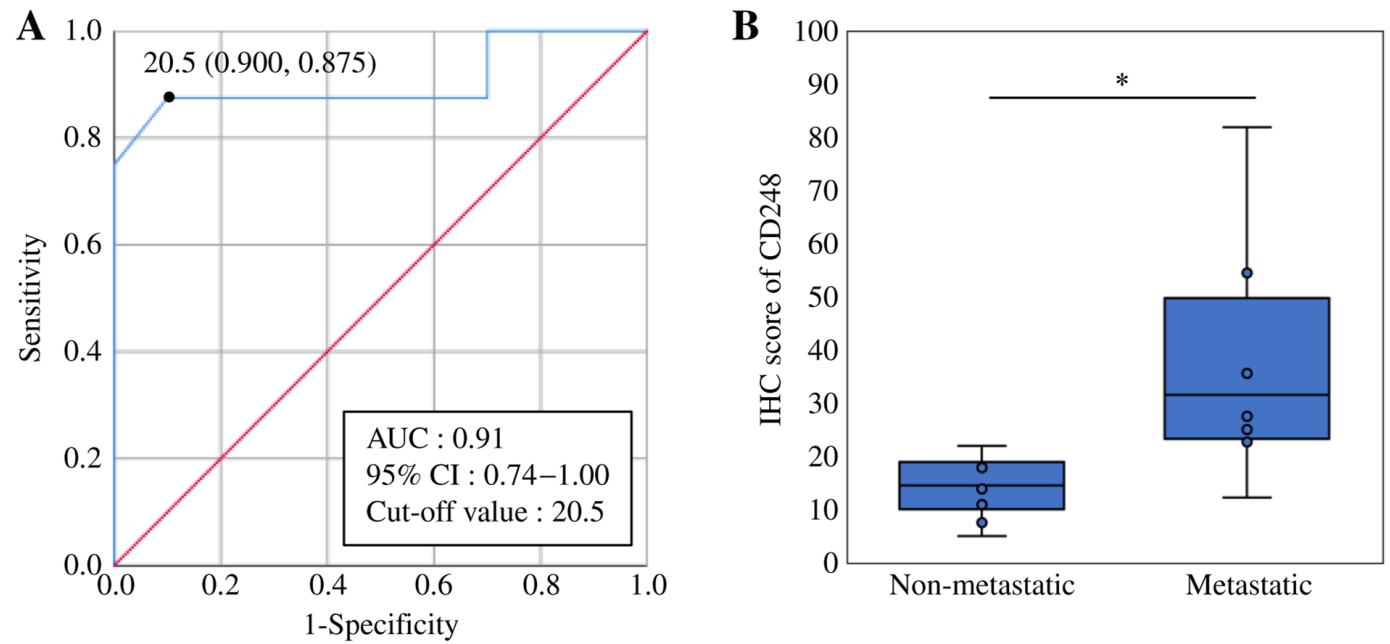

Figure 2. IHC analysis was performed to assess the association between CD248 expression and metastasis. (A) ROC curve analysis of CD248 expression. The AUC value was 0.91 (95\% CI, 0.74-1.00) and the cut-off value was 20.5. The AUC value, 95\% CI and the cut-off value were presented on the figure subpart. (B) The association between CD248 expression and metastasis. Error bars are maximum, median and minimum. "P<0.005. IHC, immunohistochemistry; ROC, receiver operating characteristic; AUC, area under the curve value; $\mathrm{CI}$, confidence interval.

The cut-off value of CD248 was 20.5, with a sensitivity of 0.875 and a specificity of 0.900 (Fig. 2A). The results demonstrated that $1 / 10$ non-metastatic OSs was positive for CD248 expression, while $7 / 8$ metastatic OSs were positive. The mean IHC score was 14.3 in the non-metastatic group (range, 5-22) and 36.9 in the metastatic group (range, 12-82). Taken together, these results suggest that CD248 is expressed at significantly high levels in OSs with metastatic disease (Fisher's exact test; $\mathrm{P}=0.003$; Fig. 2B).

Anti-endosialin antibody MORAb-004 has no cytostatic effect on OS cells. RT-qPCR analysis was performed to detect endosialin expression in OS cell lines. The expression was confirmed in all cell lines (Fig. S1). Notably, endosialin expression was observed in all cell lines used. The effect of MORAb-004 with or without FN on the viability of SaOS2, U2OS, MG63 cells was assessed in vitro. OS cells were treated with different concentrations of MORAb-004 (0, 5, 10 and $20 \mu \mathrm{mol} / \mathrm{l}$ ) and FN (0 or $10 \mu \mathrm{mol} / \mathrm{l})$. The results of the MTS assay demonstrated no significant differences in cell viability following treatment with MORAb-004, regardless of the presence of FN (Fig. 3A-C, treatment without FN; Fig. 3D and E, treatment with $\mathrm{FN}$ ).

In addition, photomicrographs of SaOS2 and U2OS cells under each condition at $\mathrm{x} 200$ magnification under an inverted microscope (ECLIPSE Ti-S100; Nikon Corporation; 

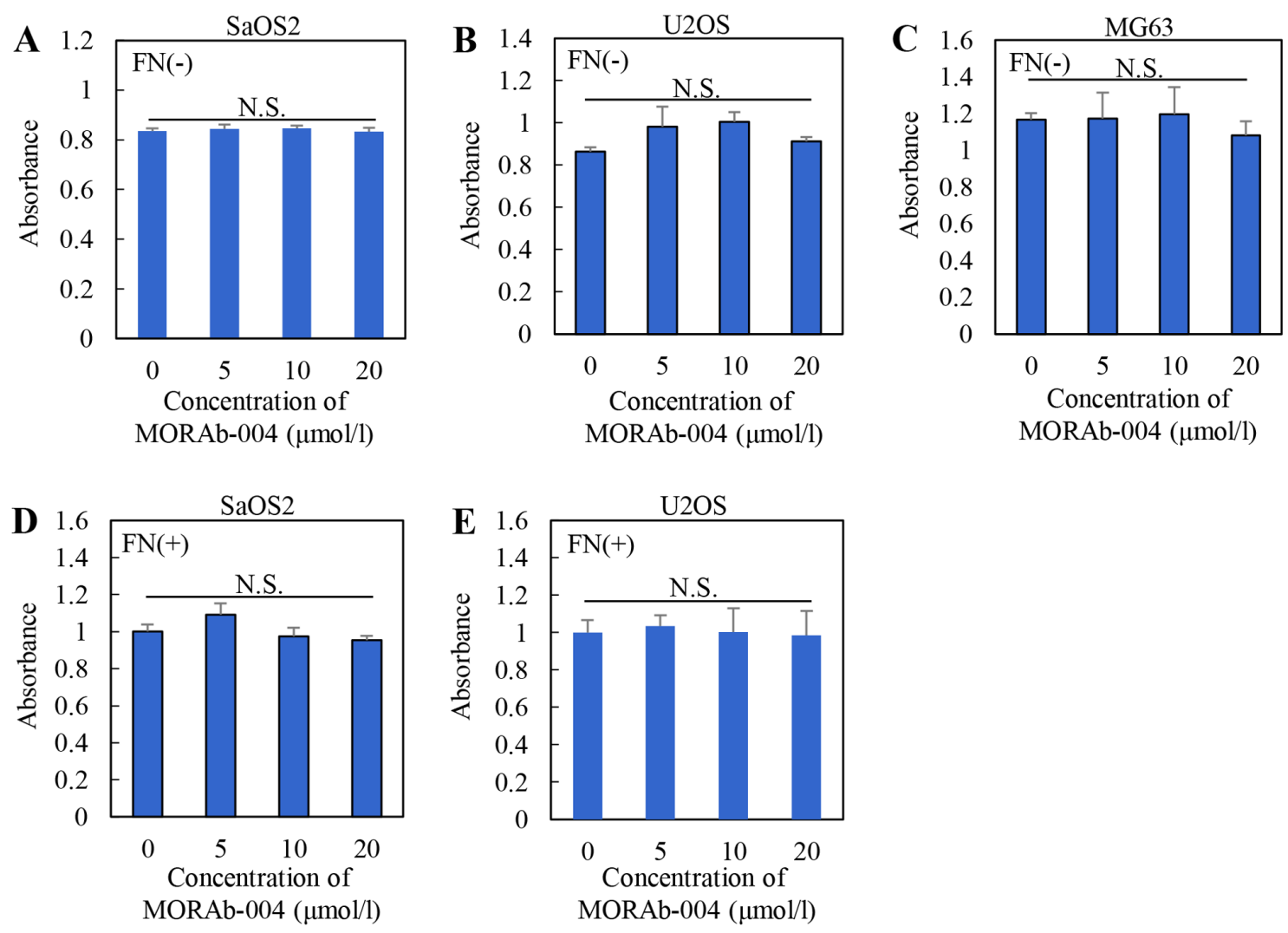

$\mathbf{F}$

Control

MORAb-004
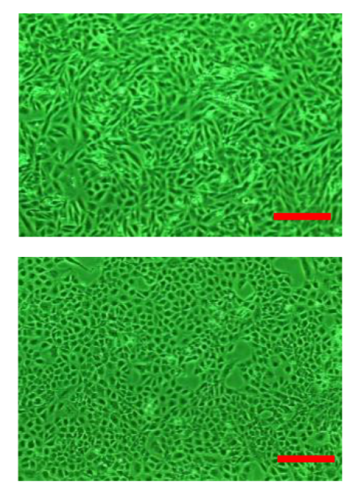

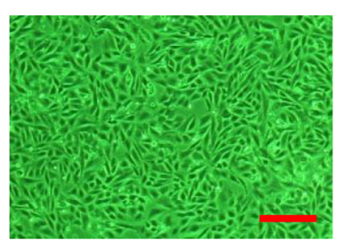

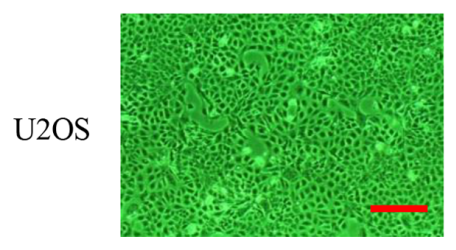

$\mathrm{SaOS} 2$
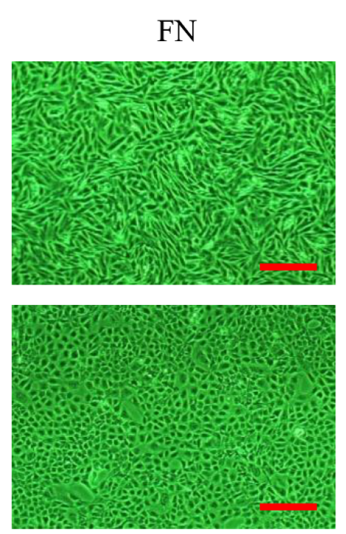

FN+MORAb-004
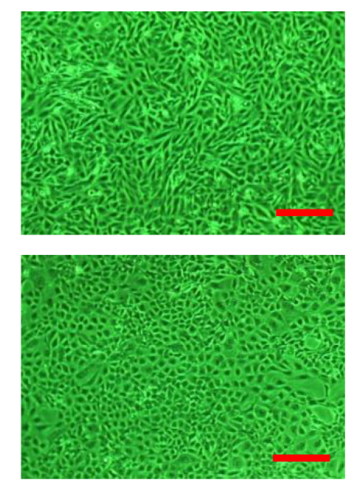

Figure 3. Effect of MORAb-004 on cell viability was assessed via the MTS assay. (A-C) SaOS2, U2OS and MG63 cells were treated without FN. (D and E) SaOS2 and U2OS cells were treated with FN. MORAb-004 had no cytostatic effect on OS cells, regardless of the presence of FN. Data are presented as the mean \pm SD. (F) Photomicrographs of SaOS2 and U2OS cells under each condition. Scale bar, $100 \mu \mathrm{m}$; magnification, x200. FN, fibronectin; OS, osteosarcoma; N.S., no significance.

https://www.microscope.healthcare.nikon.com/ja_JP/products/ inverted-microscopes/eclipse-ti-series) are presented in Fig. 3F. The actual states of cell viability in these cell lines were unaffected by MORAb-004 or FN. The effect of MORAb-004 with or without FN on the apoptosis of SaOS2 and U2OS cells was assessed (Fig. S2). No significant differences were observed in these cell lines under each condition. Collectively, these results suggest that MORAb-004 has no cytostatic effect and does not affect the apoptosis of OS cells.

MORAb-004 does not change stemness and differentiation marker expression in OS. The effect of MORAb-004 in the presence or absence of FN, one of the specific ligands for CD248 (4), on stem cell and differentiation markers was assessed via RT-qPCR analysis in the human OS cell lines,
SaOS2 and U2OS (Fig. 4A and B). Western blot analysis was also performed on the stem cell markers, CD44V9 and NS, in $\mathrm{SaOS} 2$ and U2OS cells (Fig. 4C). The results demonstrated that MORAb-004 did not change the expression of stem cell or differentiation markers.

MORAb-004 decreases OS cell migration in the presence of $F N$. To determine the efficacy of MORAb-004 in the presence of FN for sarcoma cell migration, the wound healing assay and the Transwell migration assays were performed. The results of the wound healing assay demonstrated that the migratory ability of OS cells treated with MORAb-004 alone or MORAb-004 and FN decreased in all cell lines compared with cells treated with control or only FN by the wound width measured at $24 \mathrm{~h}$ (HOS and SaOS2, $\mathrm{P}<0.001$; U2OS, $\mathrm{P}=0.010$; 


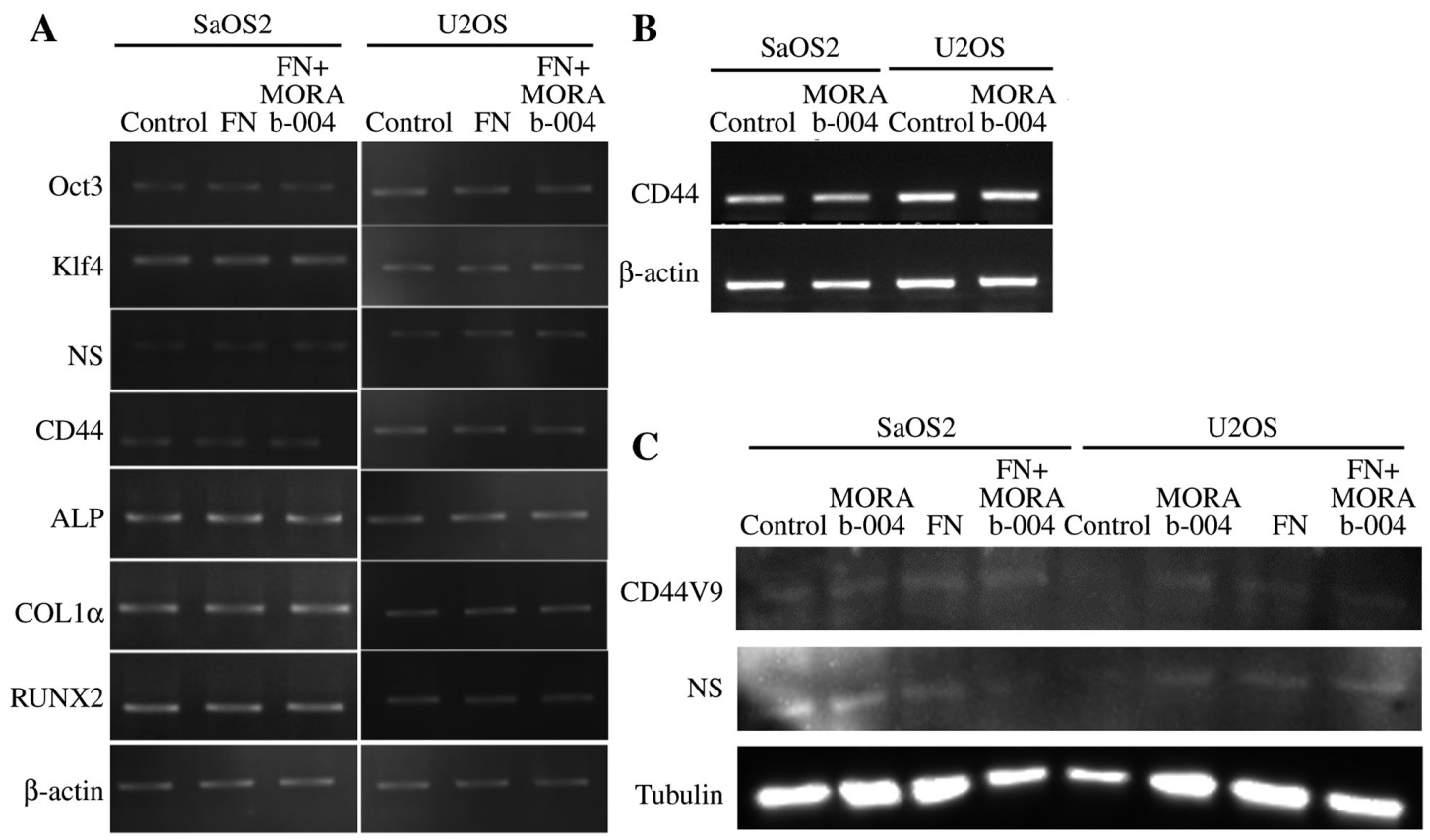

Figure 4. Effect of MORAb-004 on the expression levels of stemness and differentiation markers in OS cells. (A and B) Reverse transcription-quantitative PCR and (C) western blot analyses were performed to assess the effect of MORAb-004 on the expression levels of stem cell markers (Oct3, Klf4, NS, CD44 and CD44V9) and differentiation markers (ALP, COL1 $\alpha$ and RUNX2) in OS cell lines. OS, osteosarcoma; FN, fibronectin.

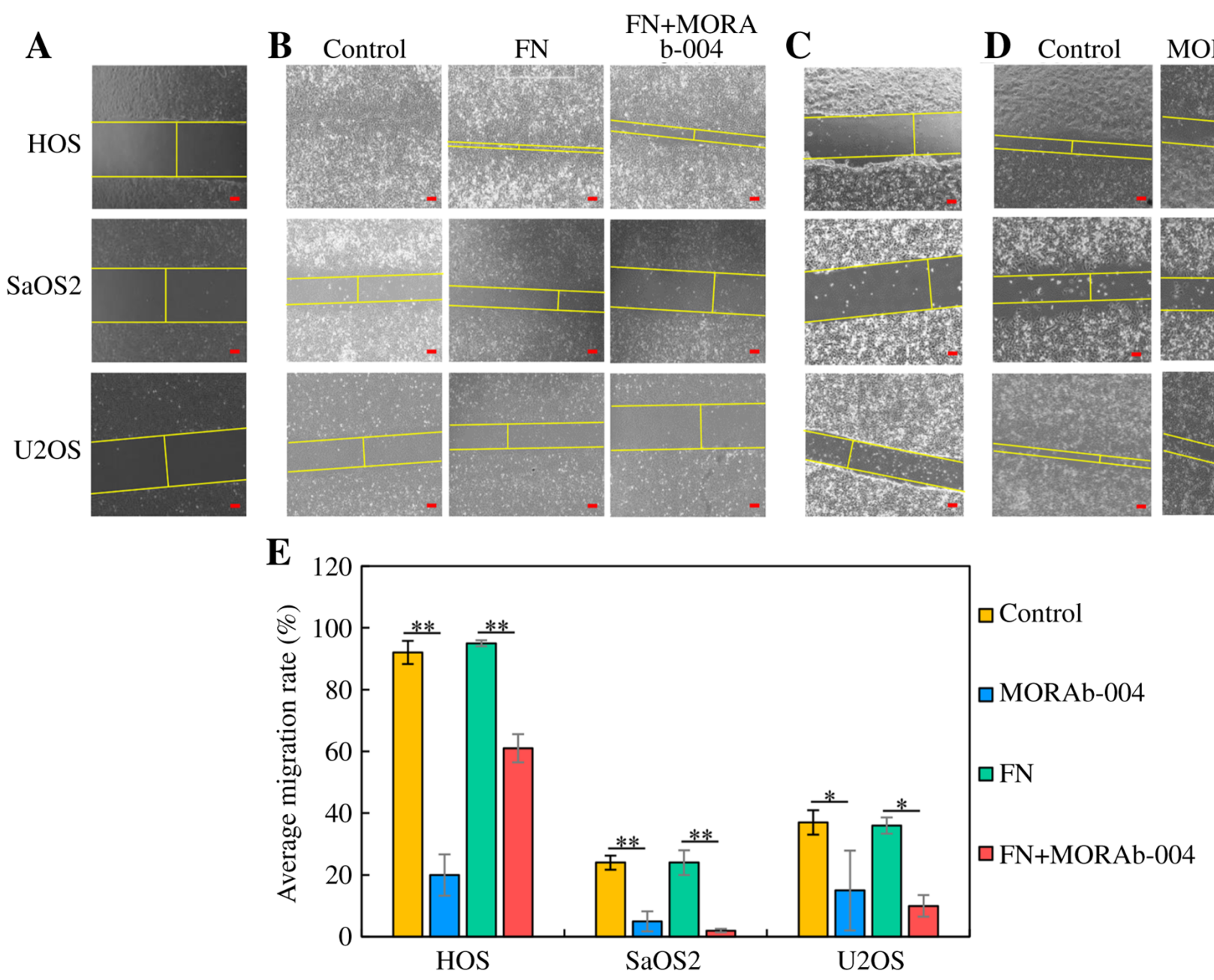

Figure 5. Efficacy of MORAb-004 with FN on the migration of HOS, SaOS2 and U2OS cells was assessed via the wound healing assay. Cells migrating into the scraped area were captured at (A and C) 0 and (B and D) $24 \mathrm{~h}$ after scraping. The wound was completely closed after $24 \mathrm{~h}$ in HOS control group. Scale bar, $200 \mu \mathrm{m}$; magnification, $\mathrm{x} 100$. (E) Average migration rate (\%) in each cell line. Data are presented as the mean $\pm \mathrm{SD}$. ${ }^{*} \mathrm{P}<0.05$. ${ }^{* *} \mathrm{P}<0.001$. FN, fibronectin. 
$\mathbf{A}$

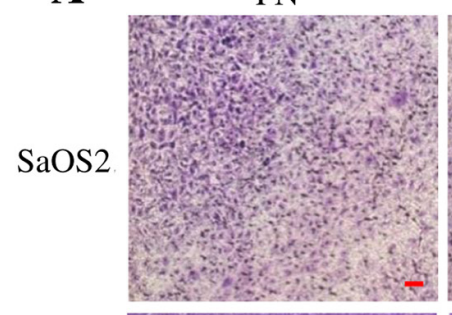

FN+MORAb-004
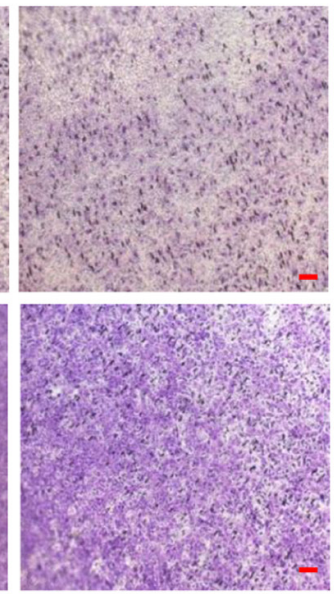

U2OS
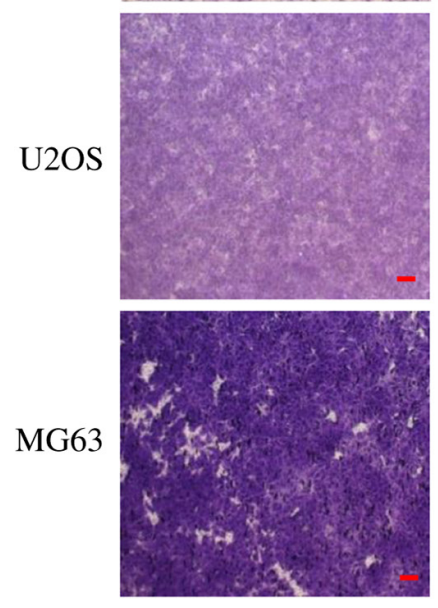

B

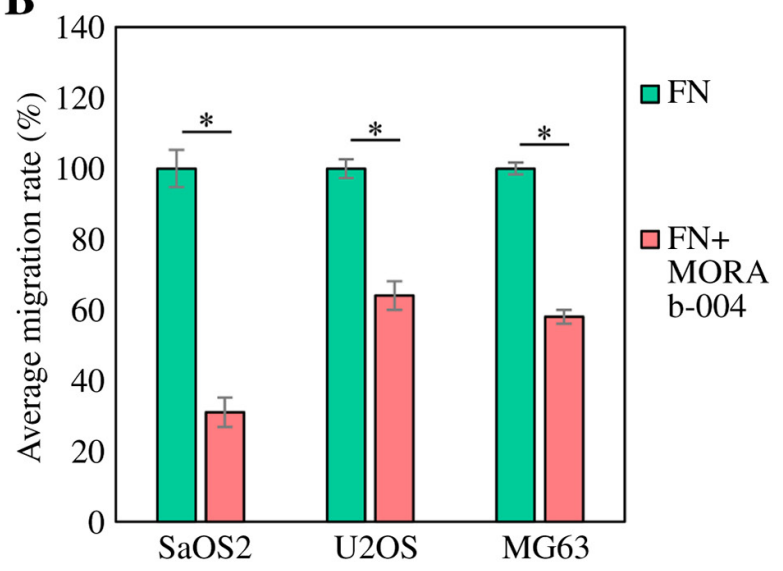

Figure 6. Efficacy of MORAb-004 with FN on the in vitro migration of $\mathrm{SaOS} 2$, U2OS and MG63 cells was assessed via the transwell migration assay. (A) Photomicrographs images of SaOS2, U2OS and MG63 cells after $24 \mathrm{~h}$. Scale bar, $200 \mu \mathrm{m}$; magnification, x100. (B) Average migration rate (\%) in each cell line. Data are presented as the mean \pm SD. ${ }^{*} \mathrm{P}<0.001$. FN, fibronectin.

Fig. 5). The width is demonstrated by yellow straight line in each figure (Fig. 5A-D). The results of the Transwell migration assay demonstrated that the number of OS cells which moved to the lower chamber also decreased following treatment with MORAb-004 (SaOS2, P=0.00066; U2OS, P=0.00089 and MG63, $\mathrm{P}=0.00003$; Fig. 6). Taken together, these results suggest that endosialin may be associated with cell migration, and MORAb-004 can suppress OS cell migration in the presence of FN.

\section{Discussion}

OS is a highly malignant bone tumor, with frequent metastasis which disseminates to the lungs and bones (19). The results of the present study demonstrated that endosialin/CD248 was highly expressed in human OS with metastatic disease; however, MORAb-004, an anti-endosialin/CD248 antibody (7), had no cytostatic effect on OS cells in vitro, and did not change the expression of stem cell and differentiation markers, suggesting that the antibody maintains their stemness property and proliferation ability. The wound healing assay and the Transwell migration assay in vitro demonstrated that Morab-004 suppressed OS cell migration in the presence of FN, suggesting that endosialin/CD248 binding to FN can promote OS cell migration.

Tumor angiogenesis is essential for tumor growth and metastasis (20). Tomkowicz et al (4) demonstrated that endosialin/CD248 mediates the proliferation of primary human pericytes via the PDGF receptor signaling pathway. In has been reported that in TEM1 knock-out mice, vessels fail to mature efficiently, which decreases the number of medium and large vessels, and increases the number of small vessels (21). This provides evidence that the endosialin/CD248-1-dependent signaling pathway controls the proliferation of human pericytes, and is required for the efficient maturation of vessels within tumors. Thus, a future strategy for suppressing tumor growth and metastasis is to target this pathway and mitigate tumor angiogenesis (22). Although blocking endosialin/CD248-mediated tumor angiogenesis would be expected to suppress tumor progression, the randomized controlled phase 2 trial of MORAb-004/ontuxizumab in combination with gemcitabine and docetaxel in advanced metastatic soft tissue sarcomas exhibited no improvement in progression-free or overall survival (9). Notably, comparative experiments with wild-type and endosialin-deficient mice revealed that stromal endosialin does not affect primary tumor growth but strongly promotes spontaneous metastasis. Mechanistically, endosialin-expressing pericytes in the primary tumor promote metastasis in a cell contact-dependent manner.

Metastasis is a multistep process, including adhesion to the extracellular matrix (ECM) (3). Tumor cells, including OS cells, adhere to matrix components via cell-surface receptors, such as integrins, which bind to the matrix protein FN (20). FN and collagen types I and IV were identified as specific ligands for endosialin/TEM-1 (4). Notably, cells expressing endosialin/TEM-1 exhibit enhanced adhesion to FN, as well as enhanced migration with increases matrix metalloproteinase- 9 activity, and these properties can be blocked by a humanized antibody directed against human endosialin/TEM1 (4).

A predictive biomarker provides information about the effect of a therapeutic intervention on clinical outcomes, and can potentially be used to select patients for therapy (9). In the present study, IHC analysis demonstrated that CD248 expression was significantly higher in metastatic OS specimens, therefore the IHC score of CD248 may be feasible as a predictor of prognosis for patients with patients. Although the detailed mechanisms of promoting invasion and metastasis through endosialin/CD248 should be elucidated in the future, it is speculated that upregulated CD248 in stromal and stem-like tumor initiating cells in OS interacts with FN and potentially activates proteases in ECM, and subsequently causes tumor microenvironment changes, which can result in tumor progression, such as invasion and metastasis (4).

Targeting endosialin/CD248 using antibodies, such as MORAb-004/ontuxizumab in combination with the conventional chemotherapeutic agents, can augment the efficacy 
against a development of metastatic lesions, potentially by reducing cell motility and suppressing invasion of OS cells. Further studies including antibody-drug conjugates are required.

The present study is not without limitations. First, the sample size for IHC was too small as biopsy specimens at a single institution were only used. Thus, clinical significance of endosialin/CD248 in sarcomas should be further evaluated using a larger sample size. In addition, the effect of MORAb-004 on OS cells was only evaluated in vitro. To verify the effect on tumor growth and metastasis, in vivo studies need to be performed in prospective studies.

In conclusion, the results of the present study demonstrated that endosialin/CD248 was highly expressed in metastatic $\mathrm{OS}$, and its binding to FN may promote OS cell migration, although did not affect tumor growth, suggesting that endosialin/CD248 may be involved in invasion and metastasis in OS. Thus, endosialin does not function as an active metastasis promoting signaling factor in inducing tumor cells to gain invasive behavior. It is expressed on the surface of tumor cells and interacts with ECM, such as FN identified as specific ligands for endosialin and changes the microenvironment. In addition, it interacts with tumor cells and endothelial cells to promote metastasis, and may be a selective therapeutic target to prevent and treat advanced diseases (3).

\section{Acknowledgements}

The authors would like to thank Ms. Mari Miyagi and Ms. Sachiyo Higashimoto for their assistance with the preparation of this manuscript. They are the secretaries of Nara Medical University Orthopedic Surgery (Nara, Japan) and contributed to the data collection and processing of clinical specimens.

\section{Funding}

The present study was partly supported by a grant to KH (grant no. 15K10455) from the Japan Society for the Promotion of Science, Chiyoda Ward (Tokyo, Japan).

\section{Availability of data and materials}

All data generated or analyzed during this study are included in this published article.

\section{Authors' contributions}

YK performed IHC analysis and prepared the manuscript. KH supervised the present study, analyzed the data and prepared the manuscript. SK and SM participated in cell culture, in vitro experiments and reverse transcription-quantitative PCR analysis. RFT helped perform the experiments and analyzed the histopathology in IHC analysis. ST and HF contributed to the acquisition of data, and the analysis and interpretation of data. HK supervised the present study and analyzed histopathology in IHC analysis. YT participated in the analysis and interpretation of clinical data for IHC, been involved in drafting and revising the manuscript with critical suggestion, given final approval of the version to be published and agreed to be accountable for all aspects of the work. $\mathrm{KH}$ and $\mathrm{HK}$ confirmed the authenticity of all the raw data. All authors have read and approved the final manuscript.

\section{Ethics approval and consent to participate}

The present study was approved by the Nara Medical University Certified Review Board (approval no. 2833; Nara, Japan) and written informed consent was provided by all patients or family members prior to the study start.

\section{Patient consent for publication}

Not applicable.

\section{Competing interests}

The authors declare that they have no competing interests.

\section{References}

1. Teicher BA: CD248: A therapeutic target in cancer and fibrotic diseases. Oncotarget 10: 993-1009, 2019.

2. Christian S, Ahorn H, Koehler A, Eisenhaber F, Rodi H-P, Garin-Chesa P,Park JE, Rettig WJ and Lenter MC: Molecular cloning and characterization of endosialin, a C-type lectin-like cell surface receptor of tumor endothelium. J Biol Chem 276: 7408-7414, 2001.

3. Viski C, König C, Kijewska M, Mogler C, Isacke CM and Augustin HG: Endosialin-Expressing Pericytes Promote Metastatic Dissemination. Cancer Res 76: 5313-5325, 2016.

4. Tomkowicz B, Rybinski K, Foley B, Ebel W, Kline B, Routhier E, Sass P, Nicolaides NC, Grasso L and Zhou Y: Interaction of endosialin/TEM1 with extracellular matrix proteins mediates cell adhesion and migration. Proc Natl Acad Sci USA 104: 17965-17970, 2007.

5. Sun D-X, Liao G-J, Liu K-G and Jian H: Endosialin expressing bone sarcoma stem like cells are highly tumor initiating and invasive. Mol Med Rep 12: 5665-5670, 2015.

6. Lange SE,Zheleznyak A, Studer M, O'Shannessy DJ, Lapi SE and Van Tine BA: Development of 89Zr-Ontuxizumab for in vivo TEM-1/endosialin PET applications. Oncotarget 7: 13082-13092, 2016.

7. Rybinski K, Imtiyaz HZ, Mittica B, Drozdowski B, Fulmer J, Furuuchi K, Fernando S, Henry M, Chao Q, Kline B, et al: Targeting endosialin/CD248 through antibody-mediated internalization results in impaired pericyte maturation and dysfunctional tumor microvasculature. Oncotarget 6: 25429-25440, 2015.

8. Diaz LA Jr, Coughlin CM, Weil SC, Fishel J, Gounder MM, Lawrence S, Azad N, O'Shannessy DJ, Grasso L, Wustner J, et al: A first-in-human phase I study of MORAb-004, a monoclonal antibody to endosialin in patients with advanced solid tumors. Clin Cancer Res 21: 1281-1288, 2015.

9. Jones RL, Chawla SP, Attia S, Schöffski P, Gelderblom H, Chmielowski B, Le Cesne A, Van Tine BA, Trent JC, Patel S, et al: A phase 1 and randomized controlled phase 2 trial of the safety and efficacy of the combination of gemcitabine and docetaxel with ontuxizumab (MORAb-004) in metastatic soft-tissue sarcomas. Cancer 125: 2445-2454, 2019.

10. Kuniyasu H, Yasui W, Shinohara H, Yano S, Ellis LM, Wilson MR, Bucana CD, Rikita T, Tahara E and Fidler IJ: Induction of angiogenesis by hyperplastic colonic mucosa adjacent to colon cancer. Am J Pathol 157: 1523-1535, 2000.

11. Bagley RG, Rouleau C, St Martin T, Boutin P, Weber W, Ruzek M, Honma N, Nacht M, Shankara S, Kataoka S, et al: Human endothelial precursor cells express tumor endothelial marker 1/endosialin/CD248. Mol Cancer Ther 7: 2536-2546, 2008.

12. Schneider CA, Rasband WS and Eliceiri KW: NIH Image to ImageJ: 25 years of image analysis. Nat Methods 9: 671-675, 2012.

13. Tanabe E, Kitayoshi M, Fujii K, Ohmori H, Luo Y, Kadochi Y, Mori S, Fujiwara R, Nishiguchi Y, Sasaki T, et al: Fatty acids inhibit anticancer effects of 5-fluorouracil in mouse cancer cell lines. Oncol Lett 14: 681-686, 2017. 
14. Li L, Qiu RL, Lin Y, Cai Y, Bian Y, Fan Y and Gao XJ: Resveratrol suppresses human cervical carcinoma cell proliferation and elevates apoptosis via the mitochondrial and p53 signaling pathways. Oncol Lett 15: 9845-9851, 2018.

15. Kishi S, Fujiwara-Tani R, Luo Y, Kawahara I, Goto K, Fujii K, Ohmori H, Nakashima C, Sasaki T and Kuniyasu H: Pro-metastatic signaling of the trans fatty acid elaidic acid is associated with lipid rafts. Oncol Lett 15: 4423-4426, 2018.

16. Luo Y, Fujii K, Ohmori H, Sasahira T, Moriwaka Y, Isobe M and Kuniyasu H: Antisense phosphorothioate oligodeoxynucleic acid for CD10 suppresses liver metastasis of colorectal cancer. Pathobiology 76: 267-273, 2009.

17. van der Meer AD, Vermeul K, Poot AA, Feijen J and Vermes I: A microfluidic wound-healing assay for quantifying endothelial cell migration. Am J Physiol Heart Circ Physiol 298: H719-H725, 2010.

18. Suarez-Arnedo A, Torres Figueroa F, Clavijo C, Arbeláez P, Cruz JC and Muñoz-Camargo C: An image J plugin for the high throughput image analysis of in vitro scratch wound healing assays. PLoS One 15: e0232565, 2020.
19. Lindsey BA, Markel JE and Kleinerman ES: Osteosarcoma Overview. Rheumatol Ther 4: 25-43, 2017.

20. Broadhead ML, Clark JCM, Myers DE, Dass CR and Choong PFM: The molecular pathogenesis of osteosarcoma: A review. Sarcoma 2011: 959248, 2011.

21. Nanda A, Karim B, Peng Z, Liu G, Qiu W, Gan C, Vogelstein B, St Croix B, Kinzler KW and Huso DL: Tumor endothelial marker 1 (Tem1) functions in the growth and progression of abdominal tumors. Proc Natl Acad Sci USA 103: 3351-3356, 2006.

22. Tomkowicz B, Rybinski K, Sebeck D, Sass P, Nicolaides NC, Grasso L and Zhou Y: Endosialin/TEM-1/CD248 regulates pericyte proliferation through PDGF receptor signaling. Cancer Biol Ther 9: 908-915, 2010 .

This work is licensed under a Creative Commons Attribution-NonCommercial-NoDerivatives 4.0 International (CC BY-NC-ND 4.0) License. 